\title{
Albucidin: a novel bleaching herbicide from Streptomyces albus subsp. chlorinus NRRL B-24108
}

\author{
Donald R Hahn, Paul R Graupner, Eleanor Chapin, John Gray, D Heim, Jeffrey R Gilbert \\ and B Clifford Gerwick
}

A novel nucleoside phytotoxin, albucidin (1), was isolated from the culture broth of Streptomyces albus subsp. chlorinus NRRL B-24108 using bioassay directed fractionation. The structure of the new natural product, albucidin, was determined by NMR and MS; however, the compound has been reported earlier in the literature following synthetic modification of oxetanocin. This is the first report of herbicidal activity for compounds of this structural type. Albucidin shows high levels of broad spectrum activity following post-emergence applications as well as moderate levels of pre-emergence activity. Accordingly, albucidin could be an important new lead for herbicide discovery.

The Journal of Antibiotics (2009) 62, 191-194; doi:10.1038/ja.2009.11; published online 27 February 2009

Keywords: albucidin; oxetanocin; phytotoxic; phytotoxin; herbicidal; Streptomyces albus

\section{INTRODUCTION}

Natural products have served as leads or starting points for the development and commercialization of numerous agricultural chemicals. In most cases, synthetic optimization has been necessary to address limitations in potency or field translation, although there are exceptions. For example, the commercial insecticide, spinosyn, is derived directly from the fermentation of Saccharopolyspora spinosa. ${ }^{1}$ However, both the strobilurin fungicides and the pyrethroid insecticides are the products of synthetic optimization of natural products with poor photostability. ${ }^{2,3}$ Particularly significant among herbicides derived from natural products are glufosinate, a phosphorylated amino acid present in the microbial tripeptides, phosalacine and bialaphos, and the triketone herbicides that were derived from the synthetic optimization of leptospermone. ${ }^{4}$ In both cases, these herbicides introduced new modes-of-action for commercial weed control, an important success factor for new products. In the course of screening for new natural product herbicides, we identified a novel phytotoxic metabolite, albucidin (1), from the culture broth of an actinomycete (Figure 1). This metabolite showed herbicidal activity against a broad spectrum of weeds. The isolation and biological activity of the metabolite as well as physiochemical properties and structural elucidation are provided in this report.

\section{RESULTS}

Fermentation and isolation

Streptomyces albus subsp. chlorinus NRRL B-24108 was fermented in shake flasks. The production of 1 began at 6 days and increased to a maximum at $12-13$ days. Greater than $90 \%$ of the phytotoxic activity in shake flask cultures was localized in the broth and could be partitioned from the aqueous broth into butanol. Isolation was achieved by semi-preparative chromatography under isocratic conditions in which the activity eluted at $6.9 \mathrm{~min}$. A strong absorbance was recorded at this retention time with absorbance maxima of 200 and $257 \mathrm{~nm}$. The total recovered yield was approximately $2 \mathrm{mgl}^{-1}$ fermentation. Interestingly, the activity eluted later under neutral $\mathrm{pH}$ conditions than under acidic conditions, which gave an early indication of the presence of an amine. The compound was not stable under acidic conditions ( $<50 \%$ recovery at $\mathrm{pH} 2,24 \mathrm{~h} ; \sim 100 \%$ recovery at $\mathrm{pH} 6.5,24 \mathrm{~h})$.

\section{Structural assignment}

The ESI LC/MS analysis of 1 indicated a molecular weight of 221, with accurate mass CI giving the molecular ion $[\mathrm{M}+\mathrm{H}]^{+}=222.0988$, $\mathrm{C}_{9} \mathrm{H}_{12} \mathrm{~N}_{5} \mathrm{O}_{2}$ requiring 222.0991. With initial NMR analysis suggesting a nucleoside, ESI LC/MS/MS was then used to probe the nature of the nucleoside base. Non-selective fragmentation of the molecule yielded an ion at $\mathrm{m} / \mathrm{z} 136$, the MS/MS spectrum of this fragment ion was acquired, and compared with that from standard samples of adenosine and 2-aminopurine riboside. The spectra for $\mathbf{1}$ and adenosine were nearly identical, whereas those from 1 and 2-aminopurine riboside gave peak intensities that were significantly different. This indicated that the position of the amino group for $\mathbf{1}$ was located at C-6'.

The NMR analysis of $\mathbf{1}$ in $\mathrm{D}_{2} \mathrm{O}$ exhibited broad signals, but revealed a nucleoside with fewer aliphatic signals than expected for adenosine. An adenine base was suggested by the two downfield signals (between 8.5 and 8.0 p.p.m.), and confirmed as described by MS. The aliphatic protons resonated as broad signals, not allowing the measurement of any coupling constants (Table 1). Analysis of an HMQC experiment 
<smiles>Nc1ncnc2c1ncn2[C]1[14CH]C([14CH2]O)O1</smiles>

Figure 1 Structure of albucidin (1).

Table 1 H NMR data for 1 and acetylated product 2

\begin{tabular}{|c|c|c|c|}
\hline Assignment & $\begin{array}{c}1 \\
\left(D_{2} O\right)\end{array}$ & $\begin{array}{c}2 \\
\left(C D_{3} O D\right)\end{array}$ & $\begin{array}{c}J(\text { for 2) } \\
(H z)\end{array}$ \\
\hline Adenine-H & 8.5 & 8.6 & - \\
\hline Adenine-H & 8.2 & 8.2 & - \\
\hline 1 & 6.6 & 6.7 & $6.9,6.9$ \\
\hline \multirow[t]{2}{*}{2} & 3.4 & 3.5 & $12.4,6.9,6.9$ \\
\hline & 3.3 & 3.3 & $12.4,6.9,6.9$ \\
\hline 3 & 4.9 & 5.0 & $\mathrm{~m}$ \\
\hline \multirow[t]{2}{*}{4} & 3.9 & 4.5 & $12.8,5.1$ \\
\hline & 3.7 & 4.4 & $12.8,2.9$ \\
\hline Acetyl Me & - & 2.2 & - \\
\hline
\end{tabular}

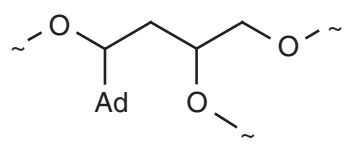

Figure 2 Identified spin system from 2D NMR spectroscopy, Ad=adenine.

indicated the presence of two methylene and two methine groups, only one methylene of which was not substituted with an oxygen atom. The molecular formula of $\mathrm{C}_{9} \mathrm{H}_{11} \mathrm{~N}_{5} \mathrm{O}_{2}$ allowed for seven unsaturations, six of which were accounted for by the adenosine base, indicating one unsaturation for this portion of the molecule, suggesting the presence of one ring, and thus one hydroxy group. Analysis of the 2D COSY spectrum indicated that the upfield methylene was indeed located between the two methines giving the spin system shown in Figure 2. With only two oxygen atoms accounted for by the molecular formula, the final ring may be assembled through ether linkages to give three possible structures for the molecule-a 3-, 4- or 5-member ring-two of which were described in the literature. Comparison with this literature data suggested that the compound contained an oxetane ring, ${ }^{5}$ rather than a furan ring ${ }^{6}$ or an epoxide.

Confirmation of this assignment came from the acetylated derivative (2), which was readily prepared from 1 with acetic anhydride in pyridine (Figure 3). Comparison of the ${ }^{1} \mathrm{H}$ NMR spectrum of 2 in $\mathrm{CDCl}_{3}$ indicated a large downfield shift for the terminal methylene protons confirming the site of the hydroxy group in albucidin, and thus the oxetane ring.<smiles>Nc1ncnc2c1ncn2[Te]1CC([14CH2]O)O1</smiles>

$1 \mathrm{R}=\mathrm{H}$

2 R=Acetyl

Figure 3 Structure of acetylated albucidin (2).

Table 2 Pre-emergence activity of $1^{a}$

\begin{tabular}{lcccc}
\hline \multirow{2}{*}{ Rate } & AVEFA & ECHCG & HELAN & IPOHE \\
\cline { 2 - 5 } g per ha & \multicolumn{4}{c}{ Visual injury (\%) } \\
\hline 2000 & 80 & 95 & 95 & 85 \\
1000 & 50 & 90 & 90 & 80 \\
500 & 45 & 60 & 80 & 80 \\
250 & 20 & 20 & 75 & 75 \\
125 & 0 & 0 & 75 & 75 \\
62.5 & 0 & 0 & 65 & 50 \\
\hline
\end{tabular}

asee text for species abbreviations.

\section{Biological activity}

Plants emerging from albucidin-treated soil (pre-emergence test) were severely stunted. Broadleaf weeds were more sensitive than grasses and most did not develop beyond the cotyledonary stage. At lower rates, the plants did develop some new growth that was bleached. The preemergence activity listed in Table 2 suggests that the mode of action is a metabolic perturbation not limited to bleaching, as most plants did not develop beyond the cotyledonary stage.

The post-emergence activity of albucidin is shown in Table 3. The onset of symptoms after post-emergence application was extremely slow, as only the new growth developed subsequent to the application initially appeared bleached. Most plants continued to grow following treatment. Pre-existing growth on the plants at the time of treatment did not show symptoms until many weeks after treatment. Symptoms progressed in severity with time, and chlorotic new growth ultimately became necrotic. Most plants treated at higher rates ( $>100 \mathrm{~g}$ per ha) died 28-35 days after application.

\section{DISCUSSION}

This is the first report of the natural product, albucidin (MW 221.0913; $\mathrm{C}_{9} \mathrm{H}_{11} \mathrm{~N}_{5} \mathrm{O}_{2}$ ), isolated from a novel strain of $S$. albus subsp. chlorinus (NRRL B-24108). The structure was discovered earlier as a semisynthetic derivative of the natural anti-viral oxetanocin from Bacillus megaterium. ${ }^{5,7}$ The $\mathrm{IC}_{50}$ of $2^{\prime}$-desmethoxy oxetanocin A (1) against HIV was approximately 10 -fold better than that of oxetanocin A. However, subsequent synthetic efforts indicated that carbocylic analogs of oxetanocin were more potent anti-viral agents than analogs with an oxetanosyl $\mathrm{N}$-glycoside; $;^{8-10}$ therefore, $\mathbf{1}$ was not investigated further. It is not known whether the anti-viral activity reported for 
Table 3 Post-emergent activity of 1, 21-day gradinga

\begin{tabular}{|c|c|c|c|c|c|}
\hline \multirow{2}{*}{$\begin{array}{l}\text { Conc. } \\
\text { (p.p.m.) }\end{array}$} & 220 & 110 & 55 & 27 & 14 \\
\hline & \multicolumn{5}{|c|}{ Visual injury (\%) } \\
\hline GOSHI & 70 & 45 & 40 & 25 & 25 \\
\hline BRSNN & 85 & 80 & 65 & 65 & 45 \\
\hline GLXMA & 80 & 60 & 60 & 60 & 45 \\
\hline BEAVA & 85 & 70 & 70 & 70 & 65 \\
\hline STEME & 90 & 75 & 70 & 50 & 45 \\
\hline XANST & 98 & 90 & 85 & 80 & 80 \\
\hline CHEAL & 75 & 55 & 65 & 50 & 40 \\
\hline IPOHE & 85 & 75 & 70 & 70 & 45 \\
\hline AMARE & 90 & 75 & 70 & 50 & 60 \\
\hline ABUTH & 85 & 80 & 75 & 65 & 55 \\
\hline VIOAR & 75 & 50 & 50 & 30 & 20 \\
\hline POLCO & 90 & 85 & 75 & 70 & 65 \\
\hline ZEAMX & 95 & 75 & 65 & 20 & 0 \\
\hline ORYSA & 65 & 55 & 40 & 60 & 35 \\
\hline TRZAX & 88 & 85 & 45 & 20 & 10 \\
\hline ALOMY & 98 & 75 & 60 & 20 & 0 \\
\hline ECHCG & 85 & 80 & 70 & 65 & 30 \\
\hline DIGSA & 90 & 75 & 65 & 65 & 60 \\
\hline SORVU & 95 & 90 & 80 & 60 & 10 \\
\hline AVEFA & 98 & 90 & 75 & 40 & 35 \\
\hline CYPES & 75 & 65 & 0 & 0 & 0 \\
\hline
\end{tabular}

asee text for species abbreviations.

oxetanocin and related compounds is linked to the herbicidal activity discovered for 1 .

Albucidin is a very potent herbicide (lethality at rates less than $100 \mathrm{~g}$ per ha in some species) that induces chlorosis and bleaching in many grass and broadleaf weeds. Although potent, the onset of symptoms after post-emergence application is very slow and effects are seen principally in new growth. The mode of action has not been pursued, but the speed of action and progression of symptoms are relatively unique and suggest that the mode of action may be new. It seems possible that the slow onset of symptoms from 1 reflects a requirement for in planta activation. Hydantocidin, a nucleoside-type phytotoxin, is phosphorylated in planta to the active inhibitor of adenylosuccinate synthase. ${ }^{11}$ Whether phosphorylation resulting in bioactivation of albucidin may similarly occur is unknown and under investigation.

\section{EXPERIMENTAL SECTION}

\section{Taxonomy of the producing organism}

The albucidin producing organism, strain LW030448, was isolated from soil at Lilly Research Laboratories in Indianapolis, IN, USA. Strain LW030448 produced well-branched vegetative mycelia and aerial hyphae, which were flexous or loose spirals. The spores were round with a smooth surface (Figure 4). Substrate mycelium was yellow to brown and aerial mycelial color was gray to green-yellow. The strain could use adonitol, inositol, mannitol and xylose for growth and could not grow at temperatures below $25^{\circ} \mathrm{C}$. The partial $16 \mathrm{~S}$ rDNA sequence (accession number DQ069278) as well as morphological and growth characteristics of strain LW030448 were highly similar to S. albus ATCC 3004. Therefore, strain LW030448 was identified as a unique subspecies, S. albus subsp. chlorinus, distinguishable by a characteristic brilliant greenyellow pigment produced in the aerial mycelium on Oatmeal Agar (ISP-3). Strain LW030448 has been deposited in the Agricultural Research Service Culture Collection at the National Center for Agricultural Utilization Research, Peoria, Illinois, USA under the accession number NRRL B-24108.

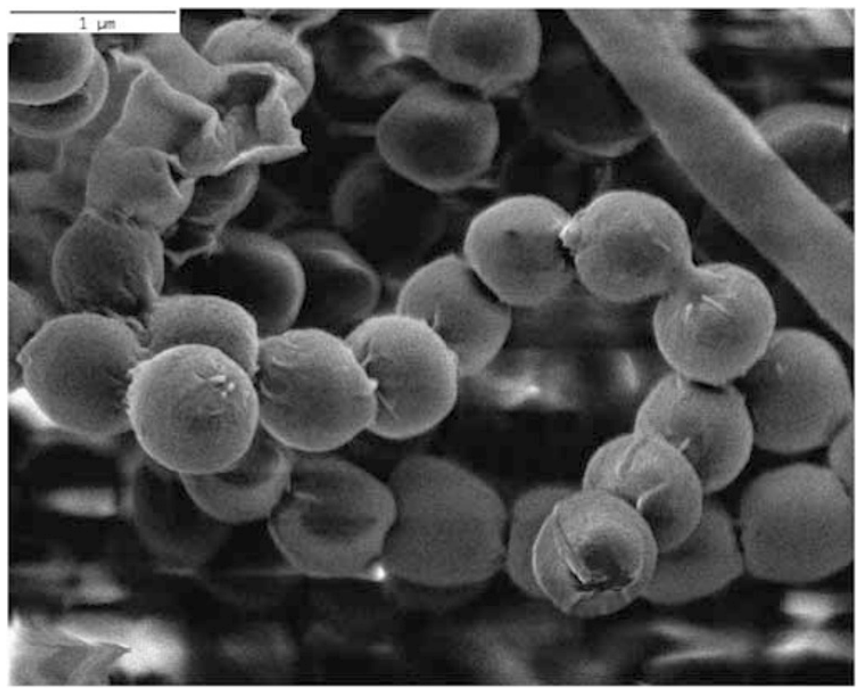

Figure 4 Scanning electron micrograph of Streptomyces albus subsp. chlorinus LW030448.

\section{Fermentation and isolation}

Strain LW030448 was fermented in Medium G $(500 \mathrm{ml}$ per 2.81-baffled Fernbach flask (Bellco, Vineland, NJ, USA)). Medium G contained soybean flour Nutrisoy (ADM, Decatur, IL, USA) $5 \mathrm{~g}$, dextrose $10 \mathrm{~g}$, glycerin $10 \mathrm{~g}$, starch solubles $5 \mathrm{~g}$, potato dextrin $20 \mathrm{~g}$, corn steep solids $5 \mathrm{~g}, \mathrm{CaCO}_{3} 3 \mathrm{~g}$, phytic acid $1 \mathrm{~g}$, cane molasses $10 \mathrm{~g}, \mathrm{FeCl}_{2} \bullet 4 \mathrm{H}_{2} \mathrm{O} 0.1 \mathrm{~g}, \mathrm{ZnCl}_{2} 0.1 \mathrm{~g}, \mathrm{MnCl}_{2} \bullet 4 \mathrm{H}_{2} \mathrm{O} 0.1 \mathrm{~g}$ and $\mathrm{MgSO}_{4} \bullet 7 \mathrm{H}_{2} \mathrm{O} 0.5 \mathrm{~g}$ in 11 of deionized water; $\mathrm{pH}$ 7.0. The flasks were inoculated with plugs from freshly sporulated agar culture and incubated at $29^{\circ} \mathrm{C}, 150$ r.p.m. for 13 days. Fermentation broth (50 l) was separated from the mycelium by centrifugation and the phytotoxic activity partitioned from the aqueous broth into butanol (1501).

Isolation was achieved by re-dissolving the dried butanol extract in $25 \%$ aqueous acetonitrile and fractionating by semi-preparative chromatography on reversed-phase C-18 (column $10 \mathrm{~mm} \times 25 \mathrm{~cm}, 5 \mu \mathrm{m}$ particle size, flow rate $5 \mathrm{ml} \mathrm{min}{ }^{-1}$ ) under isocratic conditions of $6 \%$ acetonitrile in water at $\mathrm{pH} 6.5$. The active metabolite eluted at a retention time of $6.9 \mathrm{~min}$ based on bioassay against Echinochloa crus-galli and Helianthus annuus using procedures described earlier. ${ }^{12}$

\section{NMR sectroscopy}

The NMR spectra were acquired on a Bruker $400 \mathrm{MHz}$ spectrometer (Bruker BioSpin, Billerica, MA, USA), operating at $400.13 \mathrm{MHz}$. The sample was dissolved in $0.25 \mathrm{ml}$ of $\mathrm{D}_{2} \mathrm{O}$, and placed in a $5 \mathrm{~mm}$ Shigemi micro-tube (Shigemi Inc, Allison Park, PA, USA). For the 1H/1H COSY, 256 experiments consisting of $1 \mathrm{~K}$ data points were collected with 96 transients collected per experiment. ${ }^{13} \mathrm{C}$ data were acquired using a $2 \mathrm{D}$ inverse-detected HMQC experiment. For this data set, 256 experiments were acquired over $2 \mathrm{~K}$ data points, with 160 transients per experiment.

\section{Mass spectrometry}

The LC/MS analysis was carried out using a Finnigan TSQ-700 triple quadrupole mass spectrometer (Finnigan-MAT, San Jose, CA, USA) equipped with both electrospray (ESI) and atmospheric pressure chemical oxidation (APCI) interfaces. Gradient high-performance liquid chromatography separations were accomplished on a Kromacil C- $18 \quad 4.5 \times 250 \mathrm{~mm}$ column (Eka Chemicals Separation Products, Bohus, Sweden), with simultaneous UV $(254 \mathrm{~nm})$ and mass spectral detection (positive ESI and APCI in both MS and MS/MS modes).

Accurate mass analyses were carried out using a Finnigan MAT-95q magnetic sector mass spectrometer (Finnigan-MAT) using both electron ionization, and methane chemical ionization. Samples were introduced through the direct exposure probe, and perfluorokerosene was used as an internal standard. 


\section{Acetylation of albucidin}

A very small sample $(<1 \mathrm{mg})$ of 1 was dissolved in pyridine $(0.25 \mathrm{ml})$ and acetic anhydride $(0.1 \mathrm{ml})$. The solution was stirred overnight and the excess solvents were removed by evaporation. Purification by high-performance liquid chromatography yielded the monoacetate of albucidin (2); MS (+ve ESI); 264 (100\%, M+), 136 (40\%): NMR, see Table 1.

\section{Biological activity}

Pre-emergence and post-emergent herbicide tests were conducted in a manner similar to that described earlier. ${ }^{13}$ Whole plant activity was assessed on the following species using one or both of the above means of application: Gossypium hirsutum (GOSHI), Brassica napus (BRSNN), Glycine max (GLXMA), Beta vulgaris (BEAVA), Stellaria media (STEME), Xanthium strumarium (XANST), Chenopodium album (CHEAL), Ipomoea hederacea (IPOHE), Amaranthum retroflexus (AMARE), Abutilon theophrasti (ABUTH), Viola arvensis (VIOAR), Polygonum convolvulus (POLCO), Zea mays (ZEAMX), Orysa sativa (ORYSA), Triticum aestivum (TRZAX), Alopecurus myosuroides (ALOMY), E. crus-galli (ECHCG), Digitaria sanguinalis (DIGSA), Sorghum vulgare (SORVU), Avena fatua (AVEFA), Cyperus esculentus (CYPES) and $H$. annuus (HELAN). Plants were seeded in sandy loam soil (preemergence testing) or a commercial potting mix containing $30 \%$ organic matter (post-emergence testing). For post-emergence applications, each pot was thinned to 2-25 plants per pot, depending on species. Plants were grown to a height of 3-10 cm before application. Albucidin was dissolved in methanol and diluted with $25 \%$ aqueous acetone containing $0.05 \%$ non-ionic surfactant. Serial dilutions were carried out in a solvent mixture containing $25 \%$ acetone and $0.05 \%$ non-ionic surfactant to achieve the desired p.p.m. concentrations. Each spray concentration was applied with an atomizer driven by compressed air at a pressure of $22 \mathrm{kPa}$, and approximately $1.5 \mathrm{ml}$ of total solution was applied to each pot. The plants were returned to a greenhouse (16-h photoperiod, $27^{\circ} \mathrm{C}$ day, $24^{\circ} \mathrm{C}$ night) for the duration of the study and watered by sub-irrigation. After 21 days, the treated plants were compared with untreated controls and graded on a scale of $0-100$, where 0 represented no effect and 100 indicated complete plant death.

For pre-emergence tests, albucidin was dissolved and diluted as described above and applied to the surface of pots seeded with the desired test species. Applications were made with a hand-held syringe equipped with a hollow cone nozzle to distribute the spray solution $(2.5 \mathrm{ml}$ per pot $)$ as a course mist. The pots were watered manually to move the chemical into the soil and initiate seed germination. The pots were returned to the greenhouse (16-h photoperiod, $27^{\circ} \mathrm{C}$ day, $24^{\circ} \mathrm{C}$ night) for the duration of the study and top-watered daily.
After 21 days, the emergence and height of plants from treated pots were compared with those of untreated controls on a scale of $0-100$ as described above.

\section{ACKNOWLEDGEMENTS}

We thank researchers at Eli Lilly \& Co. for the original isolation of S. albus subsp. chlorinus LW030448 from soil, Bill Heeschen of Dow, Midland, MI for the SEM micrograph and Midi Laboratories, Newark, DE for DNA sequencing.

1 Sparks, T. C., Thompson, G., Kirst, H. A., Hertlein, M. B. \& Larson, L. L. Biological activity of the spinosyns, new fermentation derived insect control agents, on tobacco budworm (Lepidoptera: Noctuidae) larvae. J. Econ. Entomol. 91, 1277-1283 (1998).

2 Sauter, H., Ammermann, E. \& Roehl, F. Stobilurins - From natural products to a new class of fungicides. in Crop Protection Agents from Nature (ed Copping, L. G.) 50-81 (The Royal Society of Chemistry, Cambridge, 1996).

3 Elliot, M. Synthetic insecticides related to the natural pyrethrins. in Crop Protection Agents from Nature (ed. Copping, L. G.) 254-300 (The Royal Society of Chemistry, Cambridge, 1996).

4 Lee, D. L. et al. The discovery and structural requirements of inhibitors of p-hydroxylphenylpyruvate dioxygenase. Weed Sci. 45, 601-609 (1997).

5 Kitagawa, M., Hasegawa, S., Saito, S., Shimada, N. \& Takita, T. Synthesis and antiviral activity of oxetanocin derivatives. Tetrahedron Lett. 32, 3531-3534 (1991).

6 Yang, T. et al. Synthesis of [4-(hydroxyl)tetrahydrofuran-2-yl]nucleosides as a novel class of uridine phosphorylase inhibitors. Tetrahedron Lett. 36, 983-986 (1995).

7 Shimada, N. et al. Oxetanocin, a novel nucleoside from bacteria. J. Antibiot. 39, 1623-1625 (1986).

8 Katagiri, N., Morishita, Y., Oosawa, I. \& Yamaguchi, M. Artificial oligonucleotides consisting of an analog of nucleoside antibiotics, carbocyclic oxetanocins. Tetrahedron Lett. 40, 6835-6840 (1999).

9 Yamaguchi, T. et al. Synthetic nucleosides and nucleotides. 43. Inhibition of vertebrate telomerases by carboxylic oxetanocin G (C.OXT-G) triphosphate analogues and influence of C.OXT-G treatment on telomere length in human HL60 cells. Nucleosides Nucleotides Nucleic Acids 25, 539-551 (2006).

10 Maruyama, T. et al. Synthesis and antiviral activity of carbocyclic oxetanocin analogues (C-OXT-A, C-OXT-G) and related compounds. II. Chem. Pharm. Bull. 41, 516-521 (1993).

11 Cseke, C. B. et al. 2a-Phosphohydantocidin: The in vivo adenylosuccinate synthase inhibitor responsible for hydantocidin phytotoxicity. Pest. Biochem. Physiol. 53, 210-217 (1996).

12 Graupner, P. R. et al. The macrocidins: novel cyclic tetramic acids with herbicidal activity produced by Phoma macrostoma. J. Nat. Prod. 66, 1558-1561 (2003).

13 Irvine, N. M. et al. Synthesis and characterization of synthetic analogs of cinacidin, a novel phytotoxin from Nectria sp. Pest. Manag. Sci. 64, 891-899 (2008). 